\title{
The Negation of Impossibility
}

\section{Abstract}

In 1951 Kenneth Arrow published a book in which he proved that social choice was impossible. There was no way to amalgamate individual preferences into a social preference in such a way that certain rational and normative conditions were met. Later Gibbard and Satterthwaite proved that any such amalgamation of individual preferences in which there was no advantage to any individual to use strategy to order their preferences insincerely in order to get a better result for themselves was impossible or led to the selection of a dictator. These impossibility theorems have been thought to rule out political direct democracy and also welfare economics giving credibility to the implication that representative democracy and capitalist economics are the best systems that can be devised.

Instead of simple amalgamation, we have devised a more general information processing system which accepts inputs from individual choosers as utilitarian ratings and outputs a social choice which can be in the form of both cardinal rating and ordinal ranking information. This system is utility based but processes the information in such a way as to alleviate concerns about interpersonal comparisons of utility. It is a hybrid utilitarian approval social choice system. Instead of the individual choosers using strategy, the system itself maximizes the efficacy of each individual input thus disincentivizing individuals from choosing insincerely. It also upgrades Arrow's five rational and normative conditions thus proving that social choice is not impossible. The result is that a utility based social choice system has been devised which negates both impossibility theorems and should give new life to welfare economics and political direct democracy. 


\section{Introduction}

In Social Choice and Individual Values, Kenneth Arrow (1951: p. 1) wrote "In a capitalist democracy there are essentially two methods by which social choices can be made: voting, typically used to make 'political' decisions, and the market mechanism, typically used to make 'economic' decisions." Initially, Arrow does not distinguish between political and economic systems claiming that both are means of formulating social decisions based on individual inputs. Arrow then purports to show that there is no rational way to make social decisions based on the amalgamation of individual ones, assuming certain rational and normative conditions are met, thus ruling out welfare economics or economic democracy and also direct political democracy. The dichotomy between political and economic systems remains with the implication that representative democracy and capitalist economics are the best systems that can be devised. Arrow's result, formerly called the paradox of voting, was first discovered by the Marquis de Condorcet (1785). Condorcet's paradox shows that majority preferences can become intransitive when there are three or more options. Arrow basically mathematized Condorcet's insight.

By contrast we develop a system for amalgamating or processing individual choices to come up with a social choice that ups the ante in terms of normative conditions and meets all of Arrow's conditions. By using preference ratings or utilities instead of preference orderings, more information is gleaned from each individual participant, and, therefore, the social choice can reflect more accurately their true preferences. For example, if one chooses between A and B using orderings, all one can say is A is preferred to B or B is preferred to A or one is indifferent between them. Instead by using preference ratings one could use a scale from 1 to 10 , for example, and rate $\mathrm{A}$ and $\mathrm{B}$ on this scale. Then there would be a substantial difference between the ratings of 10 for $\mathrm{A}$ and 1 for $\mathrm{B}$ and the ratings 6 for $\mathrm{A}$ and 
5 for B. This results in a more highly normative approach than the one Arrow uses.

Gibbard (1973) and Satterthwaite (1975) concurred with Arrow and proved that any social choice system that was strategy proof was also impossible. Gibbard stated: “An individual manipulates a system of voting if, by misrepresenting his preferences, he secures a result he prefers to the result that would obtain if he expressed his true preferences." Satterthwaite showed that the requirement for choosing procedures (what he called voting procedures) of strategyproofness and Arrow's requirements for social welfare functions are equivalent: a one-to-one correspondence exists between every strategyproof voting procedure and every social welfare function satisfying Arrow's five requirements. However, Gibbard's (1973: p. 590) results were based only on the possibility that someone could use strategy if he were astute enough to stumble on the way to do so. "Note that to call a voting scheme manipulable is not to say that, given the actual circumstances, someone is really in a position to manipulate it." Only the possibility exists in an elaborate mathematical structure. Gibbard doesn't assume that there is any actual strategy that a voter could use to manipulate the system. By contrast, we assume a situation in which an actual strategy exists which can be known both to the individual chooser and to the system which amalgamates or processes the votes/choices.

The system discussed in this paper puts individual strategy in the hands of the social choice information processing system itself so that individual choosers are disincentivized from voting insincerely. Furthermore, Gibbard's and Satterthwaite's analysis is deterministic while the problem of manipulability is inherently probabilistic. In an actual election it would be impossible for a voter to know the ideal strategy unless they knew how every other voter was going to vote. Polling, however, can provide some information of a probabilistic nature. We incorporate the fundamentally probabilistic nature of the choosing process in our analysis. The following simple example presages the path 
forward.

Let's say there are two alternatives and a number of individual choosers. Each individual chooser specifies their input as utilities on a scale which is the real line from -1 to +1 . Furthermore, let's say individual 1 has a utility of .8 for alternative A and .2 for alternative B. The strategy involved would lead individual 1 to change his sincere utility rating for alternative $\mathrm{A}$ to +1 and, similarly, candidate $\mathrm{B}$ to -1 . This would maximize the chances that A would win based just on individual 1's choice alone. However, if the information processing system, which accepts inputs from the choosers, does the strategy for them and outputs the choice as +1 for $A$ and -1 for B based on individual 1's sincere utility ratings, then there is no incentive for this individual to misrepresent their utility ratings, and they can go ahead and submit their sincere utility ratings as .8 for alternative A and .2 for alternative $\mathrm{B}$. This indicates the path forward, when more than 2 alternatives are under consideration. Of course, the chooser could misrepresent their utilities giving $\mathrm{A}+1$ and $\mathrm{B}-1$, but there would be nothing gained from doing so since the system does it for them. For more complex systems, individual choosers might actually tend to diminish their satisfaction with the outcome if they choose insincerely.

In this paper we do not dispute the Arrow and Gibbard-Satterthaite analyses lemma by lemma and proof bv proof. Their mathematics is not only impeccable, it's tautological. Instead by thinking outside the box we analyze a social choice system which accomplishes what Arrow and Gibbard-Satterthwaite purportedly set out to accomplish - a social choice system which exemplifies certain rational and normative criteria and is strategyproof. It does this in a manner that not only is more realistic in terms of actual voting/choosing systems but also exhibits a higher degree of normativeness.

A major stumbling block for the development of utilitarian social choice systems regards the issue of interpersonal comparisons. It has been thought that scales which measure the utilities of individuals are incompatible, and that any scale chosen, upon which all individuals are supposed to rate their utilities, would be arbitrary. Arrow states (1951: p. 4): "If we admit meaning to interpersonal comparisons of 
utility, then, presumably we could order social states according to the sum of utilities of individuals under each, and this is the solution of Jeremy Bentham, accepted by Edgeworth and Marshall." He (Arrow, 1951: p. 9) also states: "The viewpoint will be taken here that interpersonal comparison of utilities has no meaning and, in fact, that there is no meaning relevant to welfare comparisons in the measurability of individual utility." Thus, according to Arrow, any individual input must be based on individual preference rankings of the form $\mathrm{aPbPc} . .$. meaning $\mathrm{a}$ is preferred to $\mathrm{b}$ is preferred to $\mathrm{c}$ etc. or the notation Arrow uses $-\mathrm{aRbR}$, meaning $\mathrm{a}$ is preferred or equal to $\mathrm{b}$ etc.

Utilities for each chooser can be measured on a scale such as the real line from -1 to +1 , for example. In general there will be a utility, $u_{i}$, for each possible alternative, $c_{i}$, for each individual chooser. If $u_{a}>$ $\mathrm{ub}$, meaning the utility of alternative a is greater than the utility of alternative $\mathrm{b}$, then $\mathrm{aPb}$ and vice versa. A set of utilities will produce both preference ratings and preference orderings. We will show that, for the information processing system considered here, any affine linear transformation of an individual's set of utility ratings will yield the same output or social choice results, and, therefore, it doesn't matter which scale an individual chooses. This is not to say that the utility scale chosen by an individual is not meaningful to themselves, but just that it is meaningless in terms of their contribution to the final output of the system we analyze in this paper. To be clear we are not invoking the "one man, one vote" principle.

We develop a social choice system that is utility based, but which overcomes the objections of arbitrariness of utility scales, is strategyproof and also meets an upgraded version of Arrow's five normative and rational criteria. Therefore, social choice is not impossible, and the possibility of other such systems exists. 


\section{Utilitarian and Approval Choosing}

Utilitarian and approval choosing are exactly analogous to utilitarian voting (UV) and approval voting $(\mathrm{AV})$, and, therefore, "voting" and "choosing" are used interchangeably for the purposes of this paper. Also the words "alternative" and "candidate" will be used interchangeably. Arrow sets up the problem so that each individual chooser orders all alternatives and then society is required to come up with an ordering that is best according to his stated criteria. He states (Arrow, 1951: p. 11-12) "In the theory of consumer's choice each alternative would be a commodity bundle; ... in welfare economics, each alternative would be a distribution of commodities and labor requirements.... in the theory of elections, the alternatives are candidates." The method constructed in this paper inputs information from the individual choosers in the form of preference ratings and outputs information in the form of complete social preference rankings from which social ratings can be derived since underlying individual ratings are known. For example, if alternative $\mathrm{A}$ is one of the winners in a multi-winner election, we can compute A's average utility over the whole set of voters since we know how each voter rated A on their individual input. Summing utilities over all winners would give the social utility of a multi-winner election, for example.

Arrow's assumption of input preference orderings or rankings for each individual is a tacit assumption of equal utility scales for each individual equivalent to the "one man, one vote" principle. With the assumption that orderings represent equally spaced utilities, we can convert orderings or rankings to ratings. This may or may not be a very accurate representation of the underlying utilities, but it's the best information available if only orderings are known. For the system considered here and without loss of generality, any scale with any end points can be used for this procedure as long as the preferences orderings are equally spaced. In order to negate the Gibbard-Sattertwaite theorems, which maintain that every choosing system for which an individual chooser can use strategy to improve the outcome for 
themselves violates Arrow's conditions, we choose a social choice processing system which itself implements the optimum strategy for each individual. The system we describe here involves placing a threshold in the utility scale corresponding to an individual chooser's alternative set such that the expected value of utility of the set above threshold is maximized in the social choice outcome. All alternatives with corresponding sincere utilities above threshold are given positive approval style choices which corresponds to raising each sincere utility in that set to the maximum utility strategically. Alternatives with corresponding utilities below threshold are given negative approval style choices which corresponds to lowering each sincere utility in that set to the minimum utility strategically. This represents the optimal strategy which is implemented by the information processing system itself and not the individual chooser. Therefore, there is no incentive for an individual to use strategy or vote insincerely. Even if this is not the optimal strategy for this or any other system, to the extent that an optimal strategy is known by the individual chooser, it is also presumed to be known by the system itself. Therefore, there is no incentive for an individual to use strategy because either it would have no effect on the outcome because the system would agree with the chooser's strategy or it would have a suboptimal effect on the outcome if the system changed the chooser's strategized choice. In the latter case it would only diminish the utility of the outcome for them personally.

Claude Hillinger (2005) has made the case for utilitarian voting: "There is, however, another branch of collective choice theory, namely utilitarian collective choice, that, instead of fiddling with Arrow's axioms, challenges the very framework within which those axioms are expressed. Arrow's framework is ordinal in the sense that it assumes that only the information provided by individual orderings over the alternatives are relevant for the determination of a social ordering. Utilitarian collective choice assumes that individual preferences are given as cardinal numbers; social preference is defined as the 
sum of these numbers. The fact that voting procedures are cardinal suggests that cardinal rather than ordinal collective choice theory should be relevant."

The difference between Hillinger's statement and the system considered here is that social preference is not defined as the sum of cardinal numbers. There is a transformation from the cardinal inputs to approval style outputs which can then be converted back into cardinal numbers if desired. Hence, the system we examine is a utilitarian approval hybrid.

Hillinger (2004a) advocates Evaluative Voting (EV) in which the voter assigns a value to each candidate. For example, EV-3 assigns one of the values $(-1,0,+1)$, and then the values are summed over all candidates to determine the winner. The problem with approval voting, which Hillinger claims to ameliorate, is what to do with the candidates that are neither strongly approved of or strongly disapproved of ${ }^{\text {i.e. }}$ those in the middle. Hillinger assigns these candidates a value of zero. He (Hillinger, 2004b: pp. 1-25 ) asserts:

"Another criticism of $A V$, is due to Lawrence Ford, chair of the mathematics department, Idaho State University, ... :

One big flaw [of $A V$ ] is that most voters are fairly positive of their favorites and fairly positive of those they hate, but wishy-washy in the middle. If they choose randomly for or against approval in that middle range, the whole election can become random.

Directed against $A V$, this criticism has some validity because under $A V$, not to approve a candidate is equivalent to being against him. This puts the voter in a bind of having to be for or against, when in fact he lacks the relevant information for [such] a judgment." 
For our purposes we adopt Hillinger's EV-3 method. The use of an optimal threshold to determine which candidates get an approval style vote of +1 and which get an approval style vote of -1 clears up one of the criticisms of approval voting regarding what to do about candidates that a voter is wishy washy about. All those above threshold get a +1 vote; all those below get a -1 vote. The only ones who would get a 0 vote would be those that fell directly on the threshold.

Aki Lehtinen (2007: pp. 688-704) concludes that Arrow's Impossibility Theorem is not relevant in the final analysis: “Arrow's impossibility result and the closely related theorems given by Gibbard and Satterthwaite are unassailable as deductive proofs. However, we should not be concerned about these results because their most crucial conditions are not justifiable. Fortunately, we know that strategyproofness is usually violated under all voting rules and that IIA [Independence of Irrelevant Alternatives] does not preclude strategic voting." Contrary to Lehtinen's assertion, strategyproofness is not violated if the system itself applies the strategy instead of the individual choosers, and the system considered here does meet their "crucial conditions."

\section{Optimal Threshold Social Choice}

The Optimal Threshold Social Choice (OTSC) Information Processing System can be modeled as follows. In general we place a threshold in the scale which measures the utilities of a set of alternatives for each chooser such that the expected utility of the social choice for the set of alternatives above threshold is maximized. 


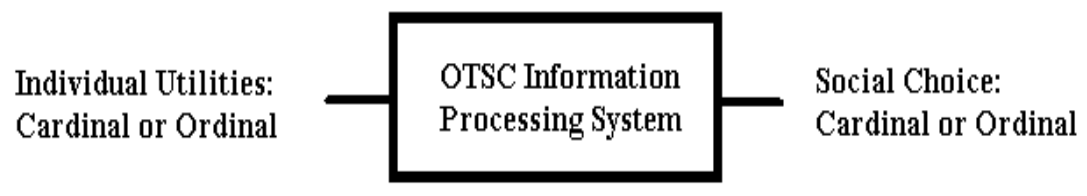

\section{Figure 1}

To state the problem formally, let S be the set of all alternatives (political candidates or workcommodity bundles or a distribution of commodities and labor requirements etc.). This set can be thought of as the nominees. For each individual chooser every alternative is associated with a utility. Let $\mathrm{W}$ be the set chosen by society based on individual inputs. $\mathrm{W} \subset \mathrm{S} .|\mathrm{W}|<|\mathrm{S}|$. We call $\mathrm{W}$ the winning set. A threshold is placed in each individual chooser's utility rating scale, which we assume, without loss of generality, to be the real line between -1 and +1 , with utilities above threshold being converted to maximum approval style choices $(+1)$ and utilities below threshold being converted to minimum approval style choices $(-1)$. The threshold $\mathrm{T}$ is a real number, $(-1<\mathrm{T}<1)$ and is placed so as to maximize the expected utility of the set $\mathrm{W}$ for each individual. In general the thresholds will be different for each individual. Summed over the individual choosers, the alternatives representing the top $|\mathrm{W}|$ approval style choices will comprise the winning set. It is assumed that each individual specifies a utilitarian style input which represents their sincere utility ratings over the set S. Later we will show that any utility scale will yield the same results so that the issue of interpersonal comparisons is moot. Therefore, all utility scales can be standardized as the real line between -1 and +1 . Also if the only information available is ordinal, it can be converted to utilitarian data before being used as inputs to the OTSC system.

To state the parameters formally for each individual: Let $\mathrm{C}$ be the set of all candidates, $\mathrm{c}_{\mathrm{i}}$ be a particular 
candidate with associated utility, $\mathrm{u}_{\mathrm{i} .} \mathrm{C}=\left\{\mathrm{c}_{1}, \mathrm{c}_{2}, \ldots \mathrm{c}_{\mathrm{i}}, \ldots \mathrm{c}_{\mathrm{n}}\right\}$ and $\mathrm{U}=\left\{\mathrm{u}_{1}, \mathrm{u}_{2}, \ldots \mathrm{u}_{\mathrm{i}}, \ldots \mathrm{u}_{\mathrm{n}}\right\}$

Let $U_{a}$ be the set of utilities above threshold and $U_{b}$ be the set of utilities below threshold. Let $C_{a}$ be the set of candidates above threshold and $\mathrm{C}_{\mathrm{b}}$ be the set of candidates below threshold. Let $\mathrm{u}_{\mathrm{a}}$ be the sum of utilities above threshold and $u_{b}$ be the sum of utilities below threshold. Let $n_{a}$ be the number of candidates above threshold and $\mathrm{n}_{\mathrm{b}}$ be the number of candidates below threshold so that $\mathrm{n}=\mathrm{n}_{\mathrm{a}}+\mathrm{n}_{\mathrm{b}}=$ total number of candidates with associated utilities. Let $\mathrm{m}=|\mathrm{W}|$, the size of the winning set.

Let $\mathrm{V}_{\mathrm{j}}$ be a random variable which represents the utility of the winning set for the $\mathrm{j}^{\text {th }}$ individual chooser, and $\mathrm{E}\left(\mathrm{V}_{\mathrm{j}}\right)$ be the expected value of this variable. Then the OTSC system maximizes

$$
\mathbf{E}\left(\mathbf{V}_{\mathrm{j}}\right)=\sum_{\mathrm{i}=1}^{\mathrm{n}_{\mathrm{a}}} \mathbf{p}_{\mathrm{i}} \mathbf{u}_{\mathrm{i}}
$$

for each individual.

In the absence of polling or probability information for each alternative, $\mathbf{p}_{\mathbf{i}}$ will be the same for each alternative. For the present we assume that polling information is unknown. The method easily extends to the case in which polling information is available. Therefore,

$$
\mathbf{E}\left(\mathbf{V}_{\mathrm{j}}\right)=\mathbf{p} \sum_{\mathrm{i}=1}^{\mathrm{n}_{\mathrm{a}}} \mathbf{u}_{\mathrm{i}}
$$

Let's identify the above expression with a ball and urn problem containing black and white balls. The white balls represent candidates above threshold and the black balls represent candidates below threshold. We posit a "picker" that picks balls randomly one at a time out of the urn without replacement and places the balls in the winning set. The mathematics for this which represent the hypergeometric distribution (Wikipedia: https://en.wikipedia.org/wiki/Hypergeometric_distribution) 
are the following:

$$
\mathbf{p}^{\prime}=\frac{\left(\begin{array}{l}
\mathbf{n}_{\mathbf{a}} \\
\mathbf{k}
\end{array}\right)\left(\begin{array}{l}
\mathbf{n}-\mathbf{n}_{\mathrm{a}} \\
\mathbf{m}-\mathbf{k}
\end{array}\right)}{\left(\begin{array}{l}
\mathbf{n} \\
\mathbf{m}
\end{array}\right)}
$$

where $\mathbf{p}^{\prime}$ equals the probability of $\mathrm{k}$ white balls placed in the winning set out of $\mathrm{m}$ picks, without replacement, from a finite population of size $n$ containing exactly $n_{a}$ white balls, wherein each draw can either produce a white ball or a black ball. Exactly which white ball (associated with a particular candidate) is picked is not known so that we use the average utility of above threshold candidates placed in the winning set for each pick. Therefore,

$$
E\left(V_{j}\right)=p^{\prime}[k=1]\left[\frac{1}{n_{a}} \sum_{i=1}^{n_{a}} u_{i}\right]+p^{\prime}[k=2]\left[\frac{2}{n_{a}} \sum_{i=1}^{n_{a}} u_{i}\right]+\ldots+p^{\prime}[k=s]\left[\frac{s}{n_{a}} \sum_{i=1}^{n_{a}} u_{i}\right]
$$

where $\mathrm{s}=\min \left\{\mathrm{m}, \mathrm{n}_{\mathrm{a}}\right\}$

Therefore,

$$
\mathbf{E}\left(\mathbf{V}_{\mathrm{j}}\right)=\sum_{\mathrm{k}=1}^{\mathrm{s}}\left\{\left[\frac{\left(\begin{array}{c}
\mathbf{n}_{\mathrm{a}} \\
\mathbf{k}
\end{array}\right)\left(\begin{array}{c}
\mathbf{n}-\mathbf{n}_{\mathrm{a}} \\
\mathbf{m}-\mathbf{k}
\end{array}\right)}{\left(\begin{array}{c}
\mathbf{n} \\
\mathbf{m}
\end{array}\right)}\right]\left[\frac{\mathbf{k}}{\mathbf{n}_{\mathrm{a}}}\right]\left[\sum_{\mathrm{i}=1}^{\mathbf{n}_{\mathrm{a}}} \mathbf{u}_{\mathrm{ij}}\right]\right\}
$$

The OTSC filter does the computations for every possible threshold to determine which threshold is best i.e. which threshold results in the maximum value of expected utility for the winning set. It does this for each chooser. All candidates above threshold will have their choices increased to +1 , and those below threshold will be decreased to -1 . Alternatives whose utilities fall exactly on the threshold will be set to zero. The results for all alternatives will then be tallied over all choosers. In addition to the individual choice thresholds there is a social choice threshold in the voting results corresponding to the 
size of the winning set. All alternatives with social choice totals above this threshold will be declared members of the winning set. Maximizing individual chooser satisfaction or utility has to do with the correct placement of the optimal threshold for each chooser.

The theory advanced here results in approval style choosing in the sense that individual cardinal inputs are converted to approval style choices. Historically, approval voting is geared to selecting one candidate from a single member district. In that case it has been shown that votes should be cast for all candidates who are above average with respect to a voter's cardinal rating scale. Smith (http://www.rangevoting.org/RVstrat3.html\#conc), proves the following: "Mean-based thresholding is optimal range-voting strategy in the limit of a large number of other voters, each random independent full-range.” Range voting is similar to utilitarian voting. Lehtinen (2007) has used expected utility maximizing voting behavior to indicate which candidates should be given an approval style vote in single member districts. He agrees with Smith that an approval style vote of +1 should be given to all candidates for whom their utility exceeds the average utility of all candidates. All others would get an $\mathrm{AV}$ vote of zero. For single member districts then, the optimal threshold is placed at the mean of the sincere ratings for each individual.

Smith and Lehtinen have shown that for a one winner outcome all ratings greater than the individual's average rating are changed to the maximum rating, and all ratings less than the average are changed to the minimum rating. Since we use maximum and minimum ratings of +1 and -1 , respectively, in our analysis, this is equivalent to placing the threshold at zero, adjusting the ratings for every candidate with a utility above that threshold to +1 and adjusting the rating for every candidate with a utility below that threshold to -1 . Preference ratings falling right on the threshold can be given a zero choice similar to Hillinger's preferred EV-3 voting method. Finally, the approval style choices for each candidate are 
summed over all choosers, and the candidate with the most approval style choices is declared the winner.

For $\mathrm{m}=1$, we have the following assuming an equal uniform distribution of utilities with $\mathrm{n}=21$ :

\section{Expected Utility vs Threshold}

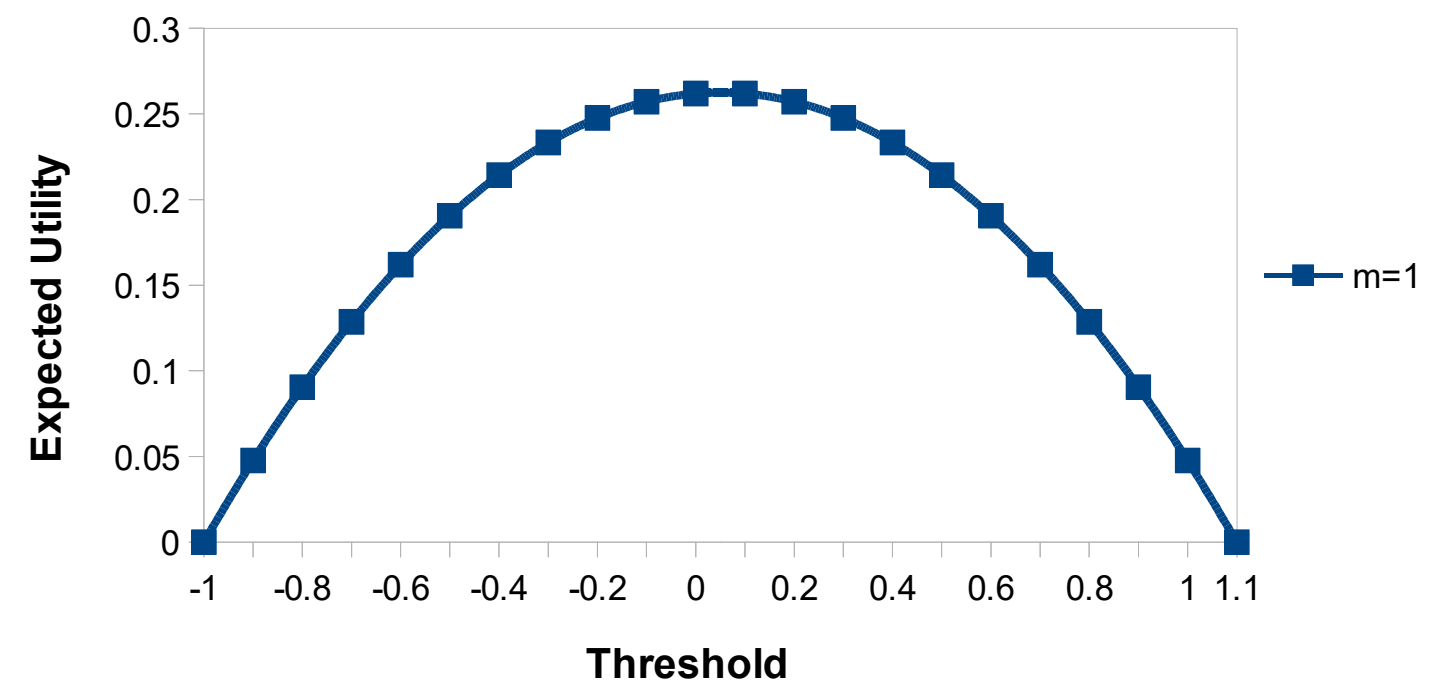

Figure 2

This example agrees with Smith ("large number of other voters, each random independent full-range") and Lehtinen when the winning set contains only one winner. Note that

$$
\lim _{n \rightarrow \infty}\left(E(1)=0 \text { and } \lim _{n \rightarrow \infty}(E(0)=E \max \right.
$$


The values for $\mathrm{m}$ greater than 1 are shown in the following figure:

\section{Expected Utility vs Threshold}

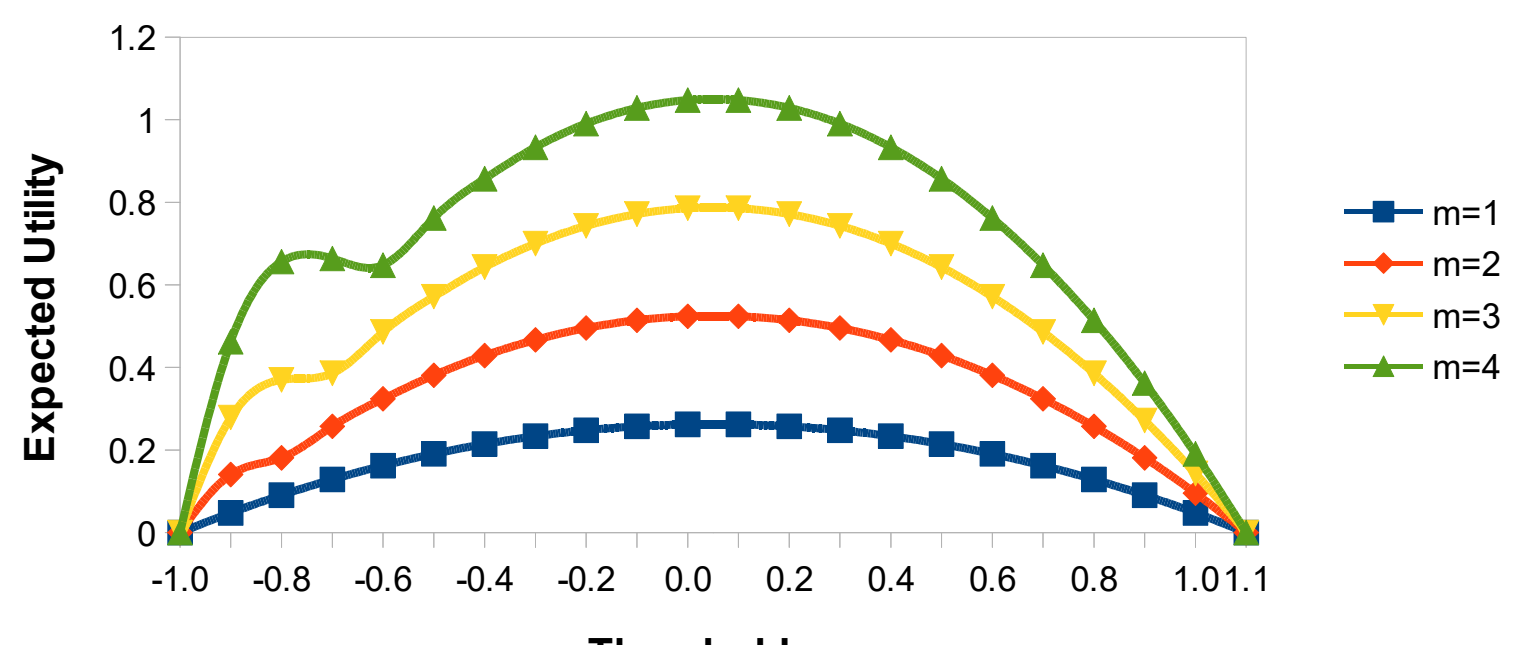

Threshold

\section{Figure 3}

The dips on the left side of the figure are due to the condition: if $(\mathbf{n}-\mathbf{n a}) \leq(\mathbf{m}-\mathbf{k})$, the probability of an above threshold candidate being chosen is 1 . Also if $\mathbf{n}_{\mathbf{a}}<\mathbf{k}$, the probability of an above threshold candidate being chosen is 0 . For more skewed distributions of utilities, the optimal threshold will not necessarily be at zero.

As an example let's assume a party is being thrown for the employees of a large company. Each employee nominates the food and drink items they would like to see available at the party. Let's say there are a total of $\boldsymbol{n}$ items nominated, but the grocery list has to be limited to $\boldsymbol{m}$ items. Optimal Threshold Social Choice can be used to determine which $\boldsymbol{m}$ items are in the winning set based on individual utilities for each item. A more elaborate example is shown in the Appendix which involves judging a skating competition. The Appendix illustrates the fact that the OTSC system resolves the intransitivities produced by Arrovian comparisons. 


\section{Optimal Threshold Social Choice Meets Arrow's Five Conditions}

Arrow's five rational and normative conditions are

1) Unrestricted domain.

2) Positive Association of Individual and Social Values

3) Independence of Irrelevant Alternatives (IIA)

4) Citizens' Sovereignty

5) Non-dictatorship

Since any alternative can be given any rating by each individual chooser, number (1) is satisfied.

Number (2) is satisfied because raising an alternative's utility in some individual's utilitarian style input from just under to just above threshold will result in that alternative's receiving one more approval style individual choice in the final summation. This would raise the social choice result by one for that alternative potentially putting that alternative in the winning set. Similarly, lowering a candidate's rating in some individual's utility scale could eliminate that alternative from the winning set. Number (4) is satisfied since the OTSC system treats all alternatives and citizens in an equal and neutral manner, and number (5) is satisfied since the winning set is based only on individual inputs in such a way that no individual has any more say over the outcome than any other individual.

As for number (3), IIA, first of all utilitarian style sincere ratings for each candidate are assumed to be independent of each other regardless of the composition of the alternative set. So if an individual rates a candidate .5 on the scale which is the real line between -1 and +1 , and then another candidate enters the race, it is assumed that the first candidate will still be rated at .5. A candidate's dropping out or entering the race is assumed not to change an individual's sincere ratings for the other candidates. Now consider the case in which, after the election occurs, a candidate dies or drops out. 
Arrow (1951: p. 26) states : "Suppose that an election is held, with a certain number of candidates in the field, each individual filing his list of preferences, and then one of the candidates dies. Surely the social choice should be made by taking each of the individual's preference lists, blotting out completely the dead candidate's name, and considering only the orderings of the remaining candidates in going through the procedure of determining a winner." Arrow implies that the voting has already occurred, but the final determination of the winner(s) has not been made. If this were the case, the OTSC Information Processing System could blot out the dead candidate's rating from all of the individual rating scales, recompute all the individual thresholds and recompute the winning set. This might have the effect of changing the composition of the winning set since all the recomputed individual thresholds may have changed.

Alternatively, let's assume that we just blot out the dead candidate's name from the final social choice. This would produce a solution which is suboptimal in the sense that recomputing the solution from scratch would produce a different final result. Even assuming that the individual thresholds are now sub-optimal, the choosers would have no incentive to cheat or strategize because they have already submitted their ballots under the assumption that the dead candidate was still in the race, and the intent of Gibbard-Sattertwaite (no insincere choosing) is still satisfied. Therefore, because of the one to one correspondence between Gibbard-Satterthwaite and Arrow, IIA is still satisfied. Certainly, the final social choice results represent a possible solution, not impossibility. If some of the dropouts were in the winning set, other candidates might be elevated to the winning set to replace them. This would not violate IIA.

Now consider the case in which a new candidate enters the race after the balloting has occurred but before the election results have been published. The added utility rating for that candidate would be 
submitted to the OTSC system by each individual chooser after the utilities for the other candidates had presumably already been submitted, and the results had already been computed. The OTSC system would then recompute the individual thresholds including the added candidate's utility rating and the final social choice results recomputed. The individual choosers would not have an incentive to rate the added candidate insincerely on their utility scales knowing that the OTSC system would give them the strategically best outcome based on the complete list of submitted utilities. Therefore, candidate add-ons would not incentivize any individual chooser to choose insincerely. Satterthwaite showed that the requirement for choosing procedures of strategyproofness and Arrow's requirements for social welfare functions are equivalent: a one-to-one correspondence exists between every strategy-proof voting procedure and every social welfare function satisfying Arrow's five requirements. A mathematical proof of this for the system under consideration is beyond the scope of this paper. Compliance with IIA is satisfied for add-ons since ratings for two candidates at a time can be uploaded for each individual chooser with thresholds recomputed at each step if necessary or as a final step thus demonstrating that the social choice can be arrived at by pairwise comparisons which Arrow's IIA demands.

Arrow (1951: p. 28) boils down IIA to pairwise comparisons: "Knowing the social choices made in pairwise comparisons in turn determines the entire social ordering and therewith the social choice function $\mathrm{C}(\mathrm{S})$ for all possible environments." If choosers built up their utilities by pairwise comparisons, the results would remain the same as if they specified all their utility inputs at the same time since sincere individual utility specifications for a particular candidate, it is assumed, do not depend on utility specifications for other candidates, and choosers know that choosing insincerely may give them a suboptimal choice in the final social choice output. 


\section{Optimal Threshold Social Choice is Strategyproof}

Since the data is processed in an optimal manner for each individual chooser by the system itself, giving each chooser the optimal strategy, the choosers have no incentive to misrepresent their preferences or to choose insincerely. They would either choose sincerely or the OTSC filter might process their input in such a way as to give them a suboptimal result. A social welfare function (Arrow's term) or a voting procedure (Satterthwaite's term) in which the strategy is inherent in the choosing procedure itself and applies to all choosers leads to a system in which there is no advantage to individuals to misrepresent either their preference orderings or preference ratings. Clearly, if the Gibbard-Satterthwaite analysis were to be extended to the probabilistic case, their theorems would not apply. The voters do not have an incentive to vote insincerely and the voting system has not led to a dictator. The strategy has been placed in the processing of the choices rather than in each individual chooser's hands. The choosers themselves are disincentivized from choosing insincerely.

The optimum strategy is to set a threshold in each individual's utilitarian style input which gives every alternative above threshold the maximum "vote" and every alternative below threshold the minimum "vote" in such a way as to maximize the expected value of utility of the social choice for each individual. This effectively turns the utilitarian style inputs into approval style outputs, but the connection with the underlying utilitarian basis of the system is maintained since the original utilities are known and can be used to compute the utility of the social choice for each individual and for society in general. Furthermore, this system resolves Arrovian intransitivities and breaks ties for the Borda system as shown in the Appendix.

\section{The Issue of Interpersonal Comparisons is Moot}

Arrow (1951: p. 10) dwells on the fact that individual utility scales are not compatible. He compares 
them with the measurement of temperature which is based on arbitrary units and the arbitrary terminal points of freezing and boiling for the Celsius scale and completely different end points for the Fahrenheit scale. "Even if, for some reason, we should admit the measurability of utility for an individual, there still remains the question of aggregating the individual utilities. At best, it is contended that, for an individual, their utility function is uniquely determined up to a linear transformation; we must still choose one out of the infinite family of indicators to represent the individual, and the values of the aggregate (say a sum) are dependent on how the choice is made for each individual. In general, there seems to be no method intrinsic to utility measurement which will make the choice compatible."

Bonner (1986) has discussed cardinal utility as follows: "Cardinal measurement is of little use in adding up social welfare if interpersonal comparisons cannot be made. ... The scale and origin of every personal index might be different, and - what is more important - any attempt to convert them to a common basis would be open to criticism." The OTSC does not "add up" or compare interpersonal utilities. Instead it processes each one in such a way that each individual's input will yield maximal results for them even though the scale and origin of every personal index may be different. Regardless of any affine linear transformation of each utility scale, the results for the OTSC process will be the same so that the individual choosers are free to choose any scale they want.

We postulate the measurability of utility for each individual. Let's say that, in general, utility can be measured as points on the real line where $-\infty<\mathbf{x}<+\infty$ and $\mathbf{x}$ is a point of the real line. It's up to the individual chooser where to place the points, including the end points, corresponding to the utilities of each candidate in the candidate set consisting of $\mathrm{n}$ alternatives, $\left\{\mathbf{c}_{1}, \mathbf{c}_{2}, \ldots, \mathbf{c}_{\mathbf{n}}\right\}$. Let's call the end points of some individual's utility scale $\mathbf{u}_{\max }$ and $\mathbf{u}_{\min }$. This will define the scale. There needs not be an actual utility assigned to either of these end points. Since the OTSC system maximizes the utility of the social 
choice for each individual, there would be an optimal threshold above which all utilities are changed to the maximum value and below which all utilities are converted to the minimum value. We maximize the individual chooser's expected utility using the equation,

$$
\mathbf{E}\left(\mathbf{V}_{\mathrm{j}}\right)=\mathbf{p} \sum_{\mathrm{i}=1}^{\mathrm{n}_{\mathrm{a}}} \mathbf{u}_{\mathrm{i}}
$$

For the OTSC system in particular, the results will be the same no matter which utility scale each individual chooses since the optimal threshold is a function of $\mathbf{n}_{\mathbf{a}}$. Let's call the value of $\mathbf{n}_{\mathbf{a}}$ associated with the optimal threshold $\mathbf{n}_{\mathbf{a}} \mathbf{o p t}$. Any affine linear transformation of a chooser's utility scale will yield the same results since $\mathbf{n}_{\mathbf{a}} \mathbf{o p t}$ will be the same point before and after the transformation. Let any individual express their utilities on a scale of their choice on the real line. For the sake of the analysis we do an affine linear transformation to convert each individual's input utility scale to one with end points -1 and $+1 . \mathbf{f}(\mathbf{u})=\mathbf{a u}+\mathbf{b}(\mathbf{a}, \mathbf{b}$ integers) represents an affine linear transformation of the individual's input scale. Let $\mathbf{f}\left(\mathbf{u}_{\max }\right)=+\mathbf{1}=\mathbf{a} \mathbf{u}_{\max }+\mathbf{b}$ and $\mathbf{f}\left(\mathbf{u}_{\min }\right)=-\mathbf{1}=\mathbf{a} \mathbf{u}_{\min }+\mathbf{b}$. It follows that $\mathbf{a}=\mathbf{2} /\left(\mathbf{u}_{\max }-\mathbf{u}_{\min }\right)$ and $\mathbf{b}=-\left(\mathbf{u}_{\max }+\mathbf{u}_{\min }\right) /\left(\mathbf{u}_{\max }-\mathbf{u}_{\min }\right)$.

There is no need to (Arrow: p. 12) "choose one out of the infinite family of indicators to represent the individual." Consequently, Arrow's statement that "the values of the aggregate are dependent on how the choice is made for each individual" is not true. Each individual makes their own choice. However, since any scale chosen by each individual will yield the same results, without loss of generality, we can standardize the choosing process by transforming individual scales to the real line between -1 and +1 before input to the OTSC system.

The OTSC procedure converts an individually specified set of utilities regardless of scale to a set of approval style decisions. The $+1 \mathrm{~s}$ represent the choices for alternatives in the alternative set; the $-1 \mathrm{~s}$ 
represent the choices against alternatives in the alternative set. This conversion is done in such a way as to maximize the power of each individual choice. Therefore, the choice made for each individual is "compatible" since it's made using the same rationale. No matter which scale an individual chooses, they have no incentive to misrepresent their true utilities.

Amartya Sen (1998) stated in his Nobel lecture “... economists came to be persuaded by arguments presented by Lionel Robbins and others (deeply influenced by "logical positivist" philosophy) that interpersonal comparisons of utility had no scientific basis. 'Every mind is inscrutable to every other mind and no common denominator of feelings is possible.' Thus, the epistemic foundations of utilitarian welfare economics were seen as incurably defective." OTSC has shown that there is a sound epistemic basis for a utility based social choice system. The OTSC system is in fact logical positivist because it has a sound scientific basis. Showing that Arrow's and Gibbard-Satterthwaite's impossibility results are invalid for just one system such as OTSC proves that social choice is not impossible potentially for other systems as well.

\section{Preference Rankings Can Be Converted to Ratings and Vice Versa}

Preference rankings can be converted to ratings for each individual which are then passed through the same OTSC procedure. Since the only information for rankings is of the form $\mathbf{a P b P c P d} \ldots$ or

aRbRcRd...), we can choose any utility scale as long as the preference rankings are equally spaced along that scale since that is the only information available. We know that the choice of which scale to use is irrelevant. Let's say we choose the real line between -1 and +1 . We let the top ranked candidate be placed at +1 and the lowest ranked candidate be placed at -1 . The other candidates then would be equally spaced on the scale. Since we assume that an optimal threshold exists, the OTSC information processing system outputs approval style positive choices for those candidates represented by utilities 
above threshold and negative choices for those candidates represented by utilities below threshold for each individual. As we have shown, any affine linear transformation of an individual's utility scale will not change the results of the OTSC processing system. The outputs are in the form of integers and represent the votes or choices for or against each alternative or candidate. Thus individual inputs can be in the form of rankings if utility information is not available. The OTSC social choice inputs and outputs can be either in the form of rankings (orderings) or in the form of ratings (utilities).

\section{Conclusions}

It has been shown that social choice is possible thus disproving the intent of both Arrow's and GibbardSatterthwaite's impossibility theorems which are in essence mathematical tautologies devoid of the inherently probabilistic nature of voting schemes and which do not assume that individual choices can be processed in any other way than simple addition. Their results apply to certain deterministic mathematical structures and were not extended to the probabilistic case we consider here. We have theoretically negated these impossibility theorems by demonstrating a system, the Optimal Threshold Social Choice (OTSC) system, which accepts Arrow's and Gibbard-Satterthwaite's conditions and yet produces actual possible results. The OTSC system accepts individual utilitarian style preference ratings as inputs and outputs approval style social choice preference rankings. It processes the inputs in such a way as to maximize the expected utility of the social choice for each individual chooser. This is done by setting a threshold in the input utilitarian data of each individual chooser and outputing positive approval style choices for those candidates above threshold and negative approval style choices for those candidates below threshold. Thus the input data is converted into approval style outputs which are then summed over all choosers. This produces social choice rankings of the alternatives. Since the OTSC system converts utilitarian style inputs to approval style outputs, OTSC is a utilitarian approval hybrid. The hybridization resolves two issues: it makes the issue of interpersonal 
comparisons moot, and it gives each chooser an optimal strategy which, when undertaken by the system itself and not by the individual chooser, disincentivizes individual choosers from choosing insincerely. Any use of strategy by individual choosers might result in a suboptimal outcome for them. Furthermore, the Gibbard-Satterthwaite results suppose that there is only the possibility that an individual chooser could strategize, not that there is an identifiable strategy that would indicate how to strategize. They also deny the inherent probabilistic nature of the choosing process which we more realistically include in our analysis.

The issue of interpersonal comparisons is moot because any affine linear transformation of an individual's utility scale will produce the same results when processed by the OTSC system. Finally, if inputs are specified as preference rankings rather than ratings, the rankings can be converted to utility style ratings which can then be processed by the OTSC system. The outputs which are in the form of social rankings can also be converted back to ratings because the underlying utility information for each individual chooser is known. Based on the social choice, utilities can be computed for each individual or for society as a whole.

Arrow's main conclusion has been known since 1785 from the work of the Marquis de Condorcet, but Arrow attempted to elaborate and recast the paradox of voting as a proof that any kind of rational system which purports to determine the public good instead leads to a dictatorship. The American and French revolutions of 1776 and 1789 respectively, although originally expressing their zeal for government by the people, ended up enshrining power in representative government precisely because the writers of their Constitutions did not trust the people. One of the most important theoreticians of the French revolution, the Abbe Sieyes, wrote (Harries-Jones, 2016), "In a country that is not a democracy - and France cannot be one - the people, I repeat, can speak or act only through its representatives." 
David Van Reybrouck writes (2016), "The French Revolution, like the American, did not dislodge the aristocracy to replace it with a democracy but rather dislodged a hereditary aristocracy to replace it with an elected aristocracy, 'une aristocratie elective', to use Rousseau's term." The impossibility theorems of Arrow and Gibbard-Satterthwaite seem to have driven this point home since they claim that direct democracy and welfare economics are impossible leaving only capitalist economics and representative democracy with a sound epistemic basis. The work presented here proves that direct political and economic democracy do in fact have a sound scientific basis and that social choice is not impossible. 


\section{Appendix}

\section{Example of Optimal Threshold Social Choice System}

Let's assume there is a figure skating competition with 9 contestants, enumerated 1 through 9 , and 5 judges alphabetized as A, B, C, D and E. The judges have to rate each contestant based on their performances. The top 3 overall will proceed to the next level of competition so the winning set will contain 3 contestants.

The expected value of utility of the winning set for each judge is the following:

$$
\mathbf{E}\left(\mathbf{V}_{\mathrm{j}}\right)=\sum_{\mathrm{k}=1}^{\mathrm{s}}\left\{\left[\frac{\left(\begin{array}{c}
\mathbf{n}_{\mathrm{a}} \\
\mathbf{k}
\end{array}\right)\left(\begin{array}{l}
\mathbf{n}-\mathbf{n}_{\mathrm{a}} \\
\mathbf{m}-\mathbf{k}
\end{array}\right)}{\left(\begin{array}{c}
\mathbf{n} \\
\mathbf{m}
\end{array}\right)}\right]\left[\frac{\mathbf{k}}{\mathbf{n}_{\mathrm{a}}}\right]\left[\sum_{\mathrm{i}=1}^{\mathbf{n}_{\mathrm{a}}} \mathbf{u}_{\mathrm{ij}}\right]\right\}
$$

$\mathrm{n}=$ number of contestants $=9$.

$\mathrm{n}_{\mathrm{a}}=$ number of contestants above threshold.

$\mathrm{m}=$ number in winning set $=3$.

$\mathrm{s}=\mathrm{n}_{\mathrm{a}}$ if $\mathrm{n}_{\mathrm{a}} \leq \mathrm{m}$ and $\mathrm{s}=\mathrm{m}$ if $\mathrm{n}_{\mathrm{a}}>\mathrm{m}$

$\mathrm{k}=$ number of above threshold candidates in winning set.

$\mathrm{u}_{\mathrm{ij}}=$ utility of contestant $\mathrm{i}$ for judge $\mathrm{j}$, a decimal value between -1 and +1 .

$V_{j}=a$ random variable representing the utility of the winning set for judge $j$.

$E\left(V_{j}\right)=$ expected value of the utility of the winning set for judge $j$.

Each judge inputs a utility rating for each contestant based on their performance as shown in Table 1. The following data was randomly generated for each judge and for each contestant. 


\begin{tabular}{|c|c|c|c|c|c|}
\hline Judge & A & B & C & D & E \\
\hline \multicolumn{6}{|l|}{ Contestant } \\
\hline 1 & -0.56 & 0.57 & -0.18 & -0.07 & 0.12 \\
\hline 2 & -0.04 & 0.11 & -0.49 & 0.41 & 0.34 \\
\hline 3 & 0.49 & 0.82 & -0.21 & -0.57 & 0.82 \\
\hline 4 & 0.77 & 0.54 & 0.33 & -0.06 & 0.21 \\
\hline 5 & -0.03 & 0.81 & 0.04 & -0.25 & -0.88 \\
\hline 6 & -0.29 & 0.45 & -0.05 & -0.93 & -0.55 \\
\hline 7 & -0.43 & -0.78 & -0.31 & -0.62 & -0.16 \\
\hline 8 & -0.37 & -0.12 & 0.14 & 0.49 & -0.67 \\
\hline 9 & 0.38 & -0.05 & -0.75 & -0.81 & -0.65 \\
\hline \multicolumn{6}{|c|}{ Table 1} \\
\hline
\end{tabular}

Table 2 shows the contestants listed from highest rated to lowest rated for each judge. Optimal thresholds were computed and are underlined.

Judge $A \quad \underline{\text { Judge } B} \quad \underline{\text { Judge } C} \quad \underline{\text { Judge } D}$ Judge $E$

Contestant Rating Contestant Rating Contestant Rating Contestant Rating Contestant Rating

$\begin{array}{llllllllll}4 & 0.77 & 3 & 0.82 & 4 & 0.33 & 8 & 0.49 & 3 & 0.82 \\ 3 & \underline{0.49} & 5 & 0.81 & 8 & 0.14 & 2 & 0.41 & 2 & 0.34 \\ 5 & -0.03 & 1 & 0.57 & 5 & \underline{0.04} & 4 & -0.06 & 4 & 0.21 \\ 2 & -0.04 & 4 & 0.54 & 6 & -0.05 & 1 & -0.07 & 1 & \underline{0.12} \\ 6 & -0.29 & 6 & 0.45 & 1 & -0.18 & 5 & -0.25 & 7 & -0.16 \\ 8 & -0.37 & 2 & 0.11 & 3 & -0.21 & 3 & -0.57 & 6 & -0.55 \\ 9 & -0.38 & 9 & -0.05 & 7 & -0.31 & 7 & -0.62 & 9 & -0.65 \\ 7 & -0.43 & 8 & -0.12 & 2 & -0.49 & 9 & -0.81 & 8 & -0.67 \\ 1 & -0.56 & 7 & -0.78 & 9 & -0.75 & 6 & -0.93 & 5 & -0.88\end{array}$

\section{$\underline{\text { Table } 2}$}


Table 3 shows the scores for each contestant and judge after all scores above threshold have been raised to +1 and all scores below threshold lowered to -1 .

\begin{tabular}{|c|c|c|c|c|c|c|c|c|c|}
\hline Judge A & & Judge B & & Judge C & & Judge D & & Judge E & \\
\hline Contestant & Rating & Contestant & Rating & Contestant & Rating & Contestant & Rating & Contestant & Rating \\
\hline 4 & 1 & 3 & 1 & 4 & 1 & 8 & 1 & 3 & 1 \\
\hline 3 & 1 & 5 & 1 & 8 & 1 & 2 & 1 & 2 & 1 \\
\hline 5 & -1 & 1 & 1 & 5 & 1 & 4 & 1 & 4 & 1 \\
\hline 2 & -1 & 4 & 1 & 6 & -1 & 1 & -1 & 1 & 1 \\
\hline 6 & -1 & 6 & 1 & 1 & -1 & 5 & -1 & 7 & -1 \\
\hline 8 & -1 & 2 & 1 & 3 & -1 & 3 & -1 & 6 & -1 \\
\hline 9 & -1 & 9 & -1 & 7 & -1 & 7 & -1 & 9 & -1 \\
\hline 7 & -1 & 8 & -1 & 2 & -1 & 9 & -1 & 8 & -1 \\
\hline 1 & -1 & 7 & -1 & 9 & -1 & 6 & -1 & 5 & -1 \\
\hline
\end{tabular}

\section{$\underline{\text { Table } 3}$}

Table 4 shows the scores for each contestant.

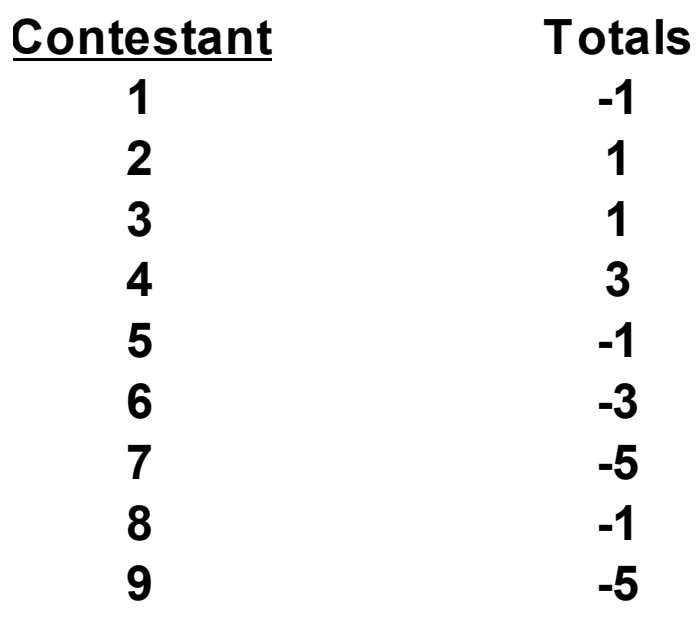

$\underline{\text { Table } 4}$ 
So the winning set is comprised of contestants 2,3 and 4 . The following are the utilities computed for each judge:

Judge A - 1.22

Judge B - 1.47

Judge C -0.37

Judge D - 0.22

Judge E - 1.37

Social Utility - 4.65

As a comparison the following table shows the Borda count for each judge and each contestant:

$\begin{array}{cccccc}\text { Contestant } & \begin{array}{c}\text { Borda Count } \\ \text { Judge A }\end{array} & \begin{array}{c}\text { Borda Count } \\ \text { Judge B }\end{array} & \begin{array}{c}\text { Borda Count } \\ \text { Judge C }\end{array} & \begin{array}{c}\text { Borda Count } \\ \text { Judge D }\end{array} & \begin{array}{c}\text { Borda Count } \\ \text { Judge E }\end{array} \\ 1 & 0 & 6 & 4 & 5 & 5 \\ 2 & 5 & 3 & 1 & 7 & 7 \\ 3 & 7 & 8 & 3 & 3 & 8 \\ 4 & 8 & 5 & 8 & 6 & 6 \\ 5 & 6 & 7 & 6 & 4 & 0 \\ 6 & 4 & 4 & 5 & 0 & 3 \\ 7 & 1 & 0 & 2 & 2 & 4 \\ 8 & 3 & 1 & 7 & 8 & 1 \\ 9 & 2 & 2 & 0 & 1 & 2\end{array}$

\section{Table 5}

Following are the totals for each contestant:

$\begin{array}{crc}\text { Contestant } & \text { Totals } & \text { Order } \\ 1 & 20 & 4 \text { tie } \\ 2 & 23 & 3 \text { tie } \\ 3 & 29 & 2 \\ 4 & 33 & 1 \\ 5 & 23 & 3 \text { tie } \\ 6 & 16 & 5 \\ 7 & 9 & 6 \\ 8 & 20 & 4 \text { tie } \\ 9 & 7 & 7\end{array}$

\section{Table 6}


The winning set is now comprised of contestants 3, 4 and a tie between contestants 2 and 5 .

Following shows a table comprised of Arrovian pairwise comparisons illustrating the intransitivity among contestants 7,8 and 9:

\begin{tabular}{|c|c|c|c|c|c|c|c|c|c|}
\hline Contestant & 1 & 2 & 3 & 4 & 5 & 6 & 7 & 8 & 9 \\
\hline 1 & & $2>1$ & $3>1$ & $4>1$ & $5=1$ & $6<1$ & $7<1$ & $8=1$ & $9<1$ \\
\hline 2 & & & $3=2$ & $4>2$ & $5<2$ & $6<2$ & $7<2$ & $8<2$ & $9<2$ \\
\hline 3 & & & & $4>3$ & $5<3$ & $6<3$ & $7<3$ & $8<3$ & $9<3$ \\
\hline 4 & & & & & $5<4$ & $6<4$ & $7<4$ & $8<4$ & $9<4$ \\
\hline 5 & & & & & & $6<5$ & $7<5$ & $8=5$ & $9<5$ \\
\hline 6 & & & & & & & $7<6$ & $8>6$ & $9<6$ \\
\hline 7 & & & & & & & & $8<7$ & $9=7$ \\
\hline 8 & & & & & & & & & $9<8$ \\
\hline 9 & & & & & & & & & \\
\hline
\end{tabular}

\section{Table 7}

The following is a comparison of the orderings for OTSC, Borda and Arrovian:

\begin{tabular}{|c|c|c|c|}
\hline $\begin{array}{l}\text { Social } \\
\text { Choice } \\
\text { Ordering }\end{array}$ & OTSC & Borda & Arrovian \\
\hline 1 & 4 & 4 & 4 \\
\hline 2 & 2,3 & 3 & 2,3 \\
\hline 3 & $1,5,8$ & 2,5 & $1,5,8$ \\
\hline 4 & 6 & 1,8 & but $6,7>8$ \\
\hline 5 & 7,9 & 6 & 9 \\
\hline 6 & & 7 & but $7=9$ \\
\hline 7 & & 9 & \\
\hline 8 & & & \\
\hline 9 & & & \\
\hline
\end{tabular}

\section{$\underline{\text { Table } 8}$}

Can the intransitivity among contestants 7,8 and 9 be resolved by referring to the underlying utilities for those contestants? The Judges' total utility scores for contestants 7,8 and 9 are $-2.30,-.53$ and -1.88 , respectively. So the ordering based on utilities would be $8>9>7$. The OTSC order is $8>7>9$ so there is 
an improvement in the sense that 8 is clearly deemed better than 7 or 9 with 7 and 9 in the last 2 places. Let's see if the underlying utilities can break ties in the winning set. Contestants 2 and 5 are tied for third. Their underlying utilities are +.33 and -.31 , respectively. These ratings could be used as a tie breaker so that third place could be given to contestant 2 . The tie between contestants 1 and 8 can also be resolved by referring to the underlying utilities which are -.12 and -.53 , respectively. Fourth place would then go to contestant 1 . 


\section{References}

1. Arrow, Kenneth J. (1951) Social Choice and Individual Values. New Haven: Yale University Press, $\mathrm{p} 1$.

2. Bonner, John (1986) Introduction to the Theory of Social Choice. Baltimore: The Johns Hopkins University Press, p. 166.

3. Condorcet, Jean-Antoine-Nicolas Caritat De (1785) Essai sur l'application de l'analyse à la probabilité des décisions rendues à la pluralité des voix. Neuilly sur Seine: Ulan Press.

4. Gibbard, A. (1973) Manipulation of voting schemes: a general result. Econometrica, 41(4): 587-601.

5. Harries-Jones, Peter (2016) Upside-Down Gods. New York City: Fordham University Press, p. 78.

6. Hillinger, Claude. (2005) The Case for Utilitarian Voting. Homo Oeconomicus 22(3): 295-321.

7. Hillinger, Claude. (2004a) Utilitarian Collective Choice and Voting. Available at http://epub.ub.uni$\underline{\text { muenchen.de/473/ }}$

8. Hillinger, Claude (2004b) Voting and the Cardinal Aggregation of Judgements. Available at: http://epub.ub.uni-muenchen.de/353/1/hillinger_voting.pdf

9. Lehtinen, Aki (2007) The Welfare Consequences of Strategic Behaviour Under Approval and Plurality Voting. European Journal of Political Economy 24(3): 688-704.

10. Satterthwaite, MA (1975) Strategy-proofness and Arrow's Conditions: Existence and Correspondence Theorems for Voting Procedures and Social Welfare Functions. Journal of Economic Theory 10(2): 187-217.

11. Sen, Amartya (1998) The Possibility of Social Choice. Nobel Lecture, Trinity College, Cambridge. Available at: https://www.nobelprize.org/uploads/2018/06/sen-lecture.pdf

12. Smith, Warren (2005) Some Theorems and Proofs. Available at: http://www.rangevoting.org/RVstrat3.html\#conc 
13. Van Reybrouck, David (2016) Against Elections. New York City: Seven Stories Press, pp. 89-91.

14. Wikipedia: Available at: https://en.wikipedia.org/wiki/Hypergeometric_distribution 